\title{
Visual Inspection System for Irregularly Formed Timing Belt with Low Reflection Ratio
}

\author{
Jaewoo Lee ${ }^{1}$ and Joongsun Yoon ${ }^{2^{*}}$ \\ ${ }^{1}$ School of Creative Science and Engineering, Waseda University \\ ${ }^{2}$ School of Mechanical Engineering, Pusan National University \\ 저반사비를 가진 비균질 타이밍 벨트를 위한 자동시각 검사시스템 \\ 이재우 ${ }^{1}$, 윤중선 ${ }^{2 *}$ \\ ${ }^{1}$ 와세다대학교 창조이공학부, ${ }^{2}$ 부산대학교 기계공학부
}

\begin{abstract}
Visual inspection systems are widely proposed for the well formed surface materials like electronics parts. But the materials with ill reflection ability have many troubles when visual inspection system is introduced. We have developed a robust visual inspection system that can work well in spite of low reflection ratio and with much noise when truth model is not known in the mixed production line. A workpiece identification technique using $\mathrm{k}$-means has been proposed to identify the type. Based on the identified type, a robust-to-noise segmentation method, called active contour, has been applied to segment the features from the image. Finally, Kalman filter has been applied to adapt the error variation. Experiment shows that performance is about to match the accuracy of manual measurement using projectors.

요 약 본 시각 검사시스템은 전자 부품과 같이 잘 형성된 표면 재료에 널리 사용되고 있다. 반사 능력이 나쁜 재 료의 경우, 시각 검사시스템이 도입될 때 많은 문제점이 발생한다. 혼합 생산 라인에서 진위의 모델을 알 수 없을 때 저 반사비와 많은 노이즈에도 잘 작동할, 강인한 시각 검사시스템을 개발하였다. 유형을 인식하기 위하여 k-means를 이용한 작업물 인식 기법이 제안되었다. 인식 유형에 기반하여 active contour라는 노이즈에 강인한 분할 기법이 영상 에서 특징을 분할하는데 응용되었다. 오차 변화를 조정하는데 Kalman 필터가 사용되었다. 자동시각 검사시스템의 실 험은 프로젝터를 이용한 수작업 측정의 정확도 수준을 보여준다.
\end{abstract}

Key Words : Visual Inspection System, Low Reflection Ratio, Active Contour Segmentation, Kalman Filter

\section{Introduction}

We propose a robust method that can identify the kind or type of timing belt automatically and measure angle of cross section of the belt with irregular surface even in the noisy situation.

Many clustering algorithms had been developed for unknown class identification [7]. Typical method such as k-means [8], E-M [1] or isodata [2] can be applied case by case. We applied k-means algorithm to reason the right model that is produced. Once the model is identified, the input data is classified by a classifier. The Baysean classifier [7] is generally utilized.

Next, feature extraction methods have been applied for extracting outline of image. Among many edge descriptors, line descriptors or curve descriptors, Hough transform [3] was not good enough because much noise in the data prevented the precise location of the boundary. As this method could be noise vulnerable, we applied the active contour, also called as a "snake" algorithm [4] with

This work was supported for two years by Pusan National University Research Grant.

*Corresponding Author : Joongsun Yoon

Tel: +82-51-510-2456 email: jsyoon@pnu.edu

Received February 16, 2012 Revised May 2, 2012 Accepted May 10, 2012 
dynamic programming [5] which maximizes the cost function. Finally, we applied Kalman filter [6] to reduce the effect of noise from the system and the environment.

\section{System Description}

The overall visual inspection system consists of the mechanical part which has mechanical positional units, the electrical part which mainly controls the mechanical device, and the computer measurement part which has image capture and processing software.

Fig. 1 shows GUI of the visual inspection system and its captured noisy image of belt section. Fig. 1(a) is the screenshot of the interface. Models of inspection are registered in advance by the operator. By running the model identification stage, the model parameters are refined to new ones. Fig. 1(b) is the raw image from the camera. This image includes much noise, which comes from the irregular surface of the belt.

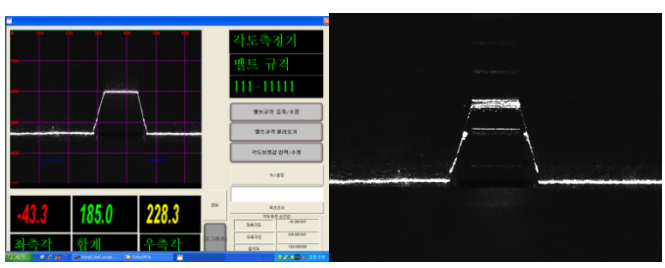

[Fig. 1] A screen-shot of the software and its input image from the camera system

\section{Visual Inspection Method}

\subsection{Model identification}

In a belt production line, various types of belts are manufactured on the conveyor lines with mixed fashion. Human inspectors check the belts whether good or not. Belts have their own criteria of quality which were determined at the stage of design. The operator in advance registers the standard parameters of the belt models with the sizes of the belt section. Then a linear classifier is applied to find the model.

In order to check the quality of all the belts that are produced on the line, each product should be compared with the criteria that are stored on the computer system.
As belts are products which are made of from the forming process, they may have variations in sizes which are different with the case of inspection of machinery parts. Therefore, we need to allow a certain level of errors and should classify one if the error level is above the threshold(s).

Moreover, belt has characteristics that its mean values may be shifted with the production unit. In order to reflect these characteristics, we use a clustering method as a method of determining the type of belt and ascertain the mean and variances of design recommendation value. We use the mean and variance of belt which were found by the clustering method when the inspection machine works.

\subsubsection{Estimation of class reference values}

A lot of samples of belt were measured in order to classify the model of belt. As a feature set for belts, we selected the longer bottom width and the height of trapezoidal section of belt. We can describe the feature vector as

$$
\mathrm{X}=\left[x_{i} \mid i=1, \ldots, n\right]
$$

Here $\mathrm{n}$ is the total number of features in the vector. As we used the bottom width and the height of section of belt, number of features in this case is 2 .

We can summarize an algorithm to identify belt types as follows:

1. Samples for every class of belts are prepared

2. We define the clustering center of $\mathrm{k}$ sets for belts as

$$
\mathbf{z}=\left\{z_{1}, z_{2}, \cdots, z_{k}\right\}
$$

where $z_{1}, z_{2}, \ldots, z_{k}$ means center values of each data sets.

Mean values of $\mathrm{k}$ sets of classes are initialized. At this time, initial value is determined by referring to the prepared data of design.

While (TRUE) \{

3. for $(\mathrm{i}=1$ to $\mathrm{m})$

4. $x_{i}$ is assigned to the class which has the nearest mean among them;

5. If (the value of assignment is the same as before) 
break;

6. For $(\mathrm{j}=1$ to $\mathrm{k})$

$z_{j}$ is substituted by the mean of sample before.

Here $\mathrm{z}_{\mathrm{j}}=\left(\mathrm{x}_{1}{ }^{\mathrm{j}}, \mathrm{x}_{2}{ }^{\mathrm{j}}\right)$ and $\mathrm{x}_{\mathrm{i}}^{\mathrm{j}}$ is $\mathrm{j}^{\text {th }}$ clustering center of $\mathrm{i}^{\text {th }}$ component of the feature vector. Also, $\mathrm{m}$ is the number of samples and $\mathrm{k}$ is the number of class of belts.

\subsubsection{Classification}

Fisher's discriminant method [8] was utilized to identify the model of input sample. Fisher's method is usually used to the classification between classes which have distinct features such as classification of machinery parts.

This method is based on the dimensional reduction by transforming the feature space to the principal space. Between-class scatter and within-class scatter are used as measures to define degree of discrimination [8].

An objective function is defined as the ratio of between-class scatter and within-class scatter. As an objective function becomes to the minimum, distance between classes is maximized. While diffusion of data in the class is minimized, transformation to the more discriminative dimension is possible.

\subsection{Robust angle measurement method}

We estimate angle by using the extracted boundary line of the cross section of belts. Error reduction method follows to decrease the minute misalignment of camera which is caused by the vibration such as air cylinder movement.

Images which come from the camera include a lot of noises, which occurs when the light of laser collides on to the irregular surfaces of the belt as shown in Fig. 1(b). In order to measure angles precisely, noises should be removed before starting to extract boundary line of cross section or robust method have to be applied. In reality, contour search algorithm and line search algorithm in the OpenCV library [9] were tested on this problem, we could not find out the exact position of the boundary due to noises. In contrast, active contour algorithm showed excellent performance to find exactly the boundary of cross section of belt even though images are contaminated with a lot of noises.

\subsubsection{Active contour algorithm}

Active contour algorithm [4] is a method to find the contour of object's boundary. This uses minimization of the cost function to find object's boundary. This algorithm assumes suitable set of points as a virtual boundary of object at the initial time. This virtual boundary is also called as "snake". The snake assumes to have energy which are the summation of several terms of energy; internal energy which is proportional to the curvature of snake at that time, image energy that attracts a snake to lines, edges and terminations and external energy that external constraint imposes.

If snake is presented as parametric form, position of points on the snake can be described as

$$
v(s)=(x(s), y(s))
$$

Here is position of the snakes.

Then, we can write the energy of snake as

$$
\begin{aligned}
E_{\text {snake }}^{*} & =\int_{0}^{1} E_{\text {snake }}(\mathbf{v}(s)) d s \\
& =\int_{0}^{1}\left\{E_{\text {int }}(\mathbf{v}(s))+E_{\text {image }}(\mathbf{v}(s))\right. \\
& \left.+E_{\text {const }}(\mathbf{v}(s))\right\} d s
\end{aligned}
$$

Here $E_{\text {int }}$ is the internal energy, $E_{\text {image }}$ is energy by image force and $E_{\text {const }}$ is energy by external constraint force.

Internal energy which curves have can be described as

$$
E_{\text {int }}=\left(\alpha(s)\left|v_{s}(s)\right|^{2}+\beta(s)\left|v_{s s}(s)\right|^{2}\right) / 2
$$

Internal energy consists of first order term which is controlled by $\alpha(s)$ and second order term which is controlled by $\beta(s)$. First order term makes curve move like membrane. Second order term makes curve move like thin-plate. $\alpha(s)$ and $\beta(s)$ are weight values, these can control importance of each terms by decreasing or increasing the values.

In order to attract snakes to the line, edge or termination in the image, energy need to be defined as

$$
E_{\text {image }}=w_{\text {line }} E_{\text {line }}+w_{\text {edge }} E_{\text {edge }}+w_{\text {term }} E_{\text {term }}
$$


$E_{\text {const }}$ means energy which comes from the external condition, this can be set to user's desired usage condition.

Active contour algorithm is a repetitive algorithm which snake at initial state moves approximately to the object's boundary through the cost function minimization. The final goal is the snake which makes cost function to be minimized at that boundary.

Algorithm moves points on the snake at each time step to the direction such a way to satisfy the gradient of energy of (4). This is the optimization process of cost function at each step. There are a variety of optimization method such as variational method [4], dynamic programming [5]. As a termination condition, if goal of energy minimization is accomplished, the snake at that time shows the boundary of the object.

\subsubsection{Extraction of boundary}

At first, as one window was established on the whole area of cross section, distortion of contour occurred due to the curvature force of the corner. Window was divided into left and right ones as independent region of interest to solve distortion at the corner. This made the extraction of boundary to almost fit to the boundary of cross section.

Internal energy of curvature uses first order term in (5). First order term means derivative of image and implies continuity of curve. Second term shows the smoothness of the curve.

The outline of the belt is not curve but has cute corner as in Fig. 1(b), $\beta(s)$ can be assumed to be zero. We define the definition of the internal energy of snake by imposing the distance between the interior points on the curve a negative sign. Then equation (7) become

$$
\begin{aligned}
E_{\text {int }}(s) & =\alpha(s)\left|\mathbf{v}_{s}(s)\right|^{2} \\
& =-|v(s+1)-v(s)|
\end{aligned}
$$

The image energy is defined as follows. To determine the cross section of the belt, it is better to detect the edges of the cross section which constitutes outer boundary.

If image energy of equation (6) is described by the energy of edge, it becomes as follows

$$
\begin{aligned}
E_{\text {iimage }} & =E_{\text {edge }} \\
& =-|\nabla I(x, y)|^{2}
\end{aligned}
$$

As the total energy of the snake constitutes as the sum of the internal energy of equation (7) and the image energy of equation (8), it becomes

$$
\begin{aligned}
& E_{\text {snake }}^{*}=\int_{0}^{1}\left\{E_{\text {int }}(v(s))+E_{\text {image }}(v(s))\right\} d s \\
& =\sum_{s=1}^{N}-|v(s+1)-v(s)|+\sum_{s=1}^{N}-|I(s+1)-I(s)|^{2}
\end{aligned}
$$

Here, $I(s)$ is the intensity at $\mathrm{s}$ on the boundary, $\mathrm{N}$ is the total number of the points on the boundary.

\subsection{Error convergence}

Fig. 2 shows the variation of measurement along the time. The reason why the measurement values are oscillating is that it is caused by the mechanical vibration.

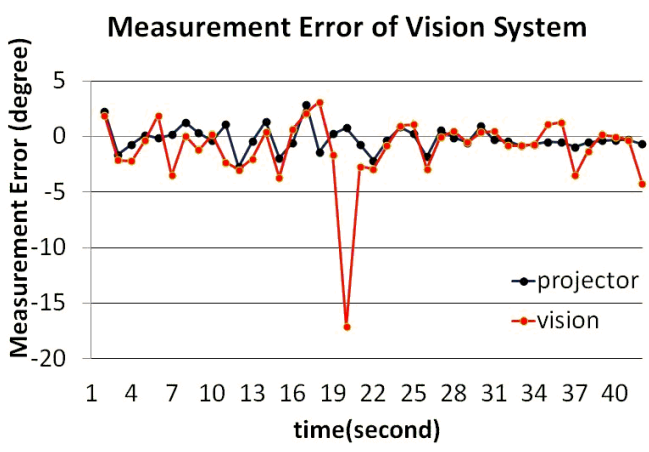

[Fig. 2] Sequence of data observation on time

Although mean values which were averaged with certain number of the result of measurement were used in order to solve this trouble, this phenomenon could not be avoided as it comes from the initial errors.

To prevent oscillation of measurement value, Kalman filter was introduced. Kalman filter reduce the error which comes from the dynamic instability of the system by using Kalman gains which is continuously improved based on the update of covariance of measurements.

\subsubsection{Kalman filter design}

Kalman filter can estimate the true values based on the measurement update on each time step. Kalman filter 
assumes linearity and Gaussian distribution on the uncertainty of the process. This assumption may work as a sort of limitation to apply this filter to the real system, because a lot of real systems are not linear, nor they don't have Gaussian distribution [6].

Kalman filter can be described as (10), (11), which consist of state equation of system and measurement equation of sensors of the system.

$$
\begin{aligned}
& \mathbf{x}_{k}=A \mathbf{x}_{k-1}+B \mathbf{u}_{k}+\mathbf{w}_{k-1} \\
& \mathbf{z}_{k}=H \mathbf{x}_{k}+\mathbf{v}_{k}
\end{aligned}
$$

Here, $\quad \mathbf{x} \in \mathfrak{R}^{n}$ is a state variable of the system, $\mathbf{z} \in \mathfrak{R}^{n}$ is the variable of measurement system. A matrix represents the system dynamics, $\mathrm{B}$ is the input gain to the system and $\mathrm{H}$ is the gain matrix showing measurement. $\mathbf{w}_{k-1}$ and $\mathbf{v}_{k}$ shows the state uncertainty and the measurement uncertainty in the system. $\quad \mathbf{w}_{k-1}$ and $\mathbf{V}_{k}$ are generally represented by the Gaussian distribution as

$$
\begin{aligned}
& p(\mathbf{w}) \sim \aleph(0, Q) \\
& p(\mathbf{v}) \sim \aleph(0, R)
\end{aligned}
$$

Here, Q means covariance of state uncertainty, R means covariance of measurement uncertainty. Kalman gain is determined by using equations (10), (11) and (12) as [6]

$$
K_{k}=P_{k}^{-} H^{T}\left(H P_{k}^{-} H^{T}+R\right)^{-1}
$$

Here, minus sign of covariance matrix $\mathrm{P}$ shows that this value was estimated based on the priori state.

Finally, a posteriori state of the system is described as

$$
\hat{\mathbf{x}}_{k}=\hat{\mathbf{x}}_{k}^{-}+K\left(\mathbf{z}_{k}-H \hat{\mathbf{x}}_{k}^{-}\right)
$$

Here $\hat{\mathbf{x}}_{k}^{-}$is the estimate of a priori state of system state, $\hat{\mathbf{x}}_{k}$ means the estimate of a posteriori state of the system.

\subsubsection{Error reduction}

In order to implement Kalman filter to the angle measurement system, true angle we want to know is regarded as state variable, measured angle which was obtained from this system is regarded as measurement variable in the Kalman filter.

If we assume that there is no input and state noise has Gaussian distribution which has mean of 0 , variance of $1 \%$, the state equation of the system is

$$
x_{k}=x_{k-1}+N(0,0.01)
$$

Moreover, we assume that measurement gain is 1 and the noise induced from the system has mean of 0 , variance of $0.1 \%$. Then measurement equation becomes

$$
z_{k}=x_{k}+N(0,0.01)
$$

\section{Experimental Results}

Experiments were carried out for three types of model which are $18^{\circ}, 19^{\circ}$ 도 and $20^{\circ}$.

We used the bottom width and the height of belt as a feature set for classification. Three types of belt could be identified by the k-means method. The result was converged within 10 recursive cycles.

As can be seen from Fig. 3 the result from the dynamic programming was excellent to extract the center line of the image on noise. It was robust to many outliers in this case. And the error reduction using Kalman filter also showed the good performance compared to the manual projector measurement.

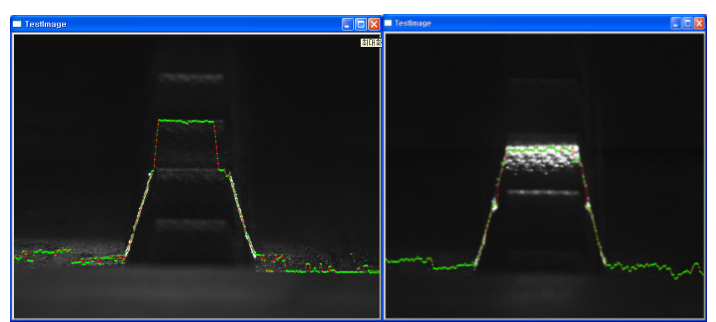

(a)

(b)

[Fig. 3] Extraction of outline boundary of the cross section of the belt. (a) mismatch in case of worst reflection (b) excellent match even in the big noise in the roof of the cross section

Fig. 4 shows that the application of filter was effective to let the data sequence converge to the admissible range of error. As we can see from Fig. 4, the error is depressed by the effect of the Kalman filtering. 


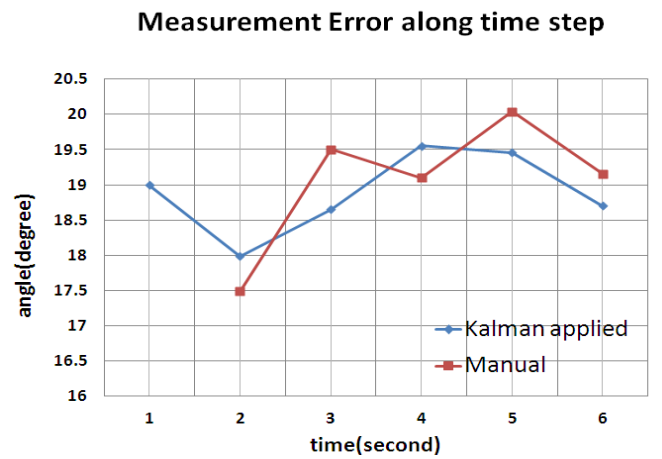

[Fig. 4] Comparison between the projected data("manual" means operator checked) and the automatic inspection data(with Kalman filtering applied)

\section{Conclusion}

We have developed a robust visual inspection system in a mixed production line with materials having low reflection ratio. The autonomous model identification and its parameter update of classifier were accomplished. The extraction of boundary line of cross section of the belts proved to be robust in spite of a lot of noise inclusions. Errors from the unstable mechanical system could be reduced reasonable level by using the Kalman gain update. This has been proved to be good to use in the well illuminated, noisy environment like a rubber belt production line.

\section{References}

[1] A. P. Dempster, N. M. Laird and D.B. Rubin, "Maximum Likelihood Estimation from Incomplete Data via the EM Algorithm", Journal of Royal Statistical Society, vol. 39, no. 1, pp. 1-38, 1977.

[2] H. Gu, G.-D. Su, C. Du, "Fuzzy and ISODATA Classification of Face Contours", Proceedings of International Conference of Machine Learning and Cybernetics, 26-29, August, 2004.

[3] R. O. Duda and P. E. Hart, "Use of the Hough Transform to Detect Lines and Curves in Pictures", Comm. ACM, vol. 15, no. 1, pp. 11-15, Jan. 1972.

[4] M. Kass, A. Witkin, and D. Terzopoulos, "Snakes: Active Contour Models", International Conference on Computer Vision, pp. 259-268, 1987.
[5] A. A. Amini, T. E. Weymouth, and R. C. Jain, "Using Dynamic Programming for Solving Variational Problems in Vision", IEEE Trans. Pattern Analysis and Machine Intelligence, vol. 12, no. 9, pp. 855-867, 1990.

[6] S. J. Julier and J. K. Uhlmann, "A New Extension of the Kalman Filter to Nonlinear Systems", Proceedings of International Symposium on Aerospace/Defense Sensing, Simulation and Controls, 1997.

[7] R. O. Duda, P. E. Hart and D. G. Stork, Pattern Recognition, 2nd Edition, Wiley and Sons, pp. 27-30, 2000.

[8] C. M. Bishop, Pattern Recognition and Machine Learning, Springer, 2009.

[9] G. Bradsky and A. Kaehler, Learning OpenCV: Computer Vision with the OpenCV Library, O'Reilly Media, 1st edition, 2008.

\section{Jaewoo Lee}

[Regular member]

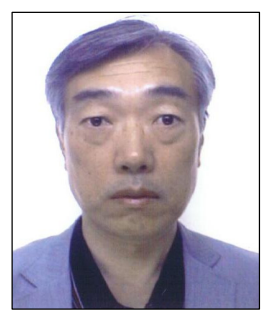

- Feb. 1883 : Pusan National Univ., BS

- Feb. 1988 : KAIST, MS

- 2009-Current : Waseda Univ., PhD

- Feb. $1988 \sim$ Dec. 1992 : Samsung Electronics, Engineer

- Feb. $1994 \sim$ Dec. 1999 :

Hyundai Heavy Industry, Manager

- Mar. 2000 Jul. 2007 : NeuroSystems, CEO

$<$ Research Interests $>$

Biorobotics, Sensor Network, Medical Imaging

Joongsun Yoon

[Regular member]

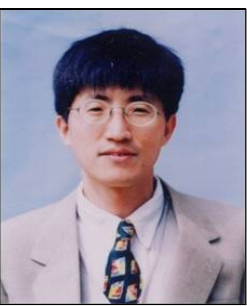

- Feb. 1981 : Seoul National Univ., BS

- Feb. 1983 : Seoul National Univ., MS

- OCt. 1988 : Lehigh Univ., PhD

- Aug. 1989 Jul. 1991 :

Samgsung Electronics, Senior Researcher

- Jul. 1991 Jul. 1993 : Kumoh National Univ., Dept. of Precision Mechanical Eng., Professor

- Jul. 1993 current : Pusan National Univ., School of Mechanical Eng., Professor

$<$ Research Interests $>$

Robotics, Automation, HCI 